\title{
Proyectos de Gran Escala, Género y Relocalizaciones Urbanas. Ciudad de Federación (Argentina)*
}

\author{
María Rosa Catullo**
}

\section{Resumen}

Los Proyectos de Gran Escala, como son las represas hidroeléctricas, presentan una serie de consecuencias entre las cuales una de las más importantes es la relocalización compulsiva de población. A través de más de 30 años hemos investigado el proceso de relocalización de la ciudad de Federación (Argentina), consecuencia de la construcción de la represa binacional argentino-uruguaya de Salto Grande. En este artículo analizamos la forma desigual en que fueron afectadas las mujeres federaenses durante dicho proceso relocalizatorio, focalizando las relaciones género-clases sociales y género-edad.

Palabras clave: Género, Proyectos de Gran Escala, Relocalizaciones urbanas, Federación, Argentina.

\footnotetext{
" Recibido el 24 de agosto de 2017, aceptado el 28 de agosto de 2018.

** Antropóloga Social. Investigadora Independiente del Consejo Nacional de Investigaciones Científicas y Técnicas (CONICET), La Plata, Argentina. mcatullo2000@yahoo.com.ar
} 
Large Scale Projects, Gender and Urban Resettlements. City of Federación (Argentina)

\begin{abstract}
Large Scale Projects, such as hydroelectric dams, have a number of consequences, one of the most important of which is compulsory population relocation. Over the course of more than 30 years we have investigated the relocation process of the city of Federación (Argentina), as a consequence of the construction of the Argentine-Uruguayan bi-national dam of Salto Grande. In this article, we analyze the unequal way in which the women of Federación were affected during this relocation process, focusing on the gender-social class and the gender-age relationships.
\end{abstract}

Keywords: Gender, Large Scale Project, Urban Resettlements, city of Federación, Argentina. 


\section{Introducción}

Los estudios sobre los impactos socioambientales resultado de Proyectos en Gran Escala, tales como represas, puentes, ciudades, son estudiados por los antropólogos sociales desde hace ya varias décadas, cobrando mayor vigencia en los países del Mercosur a partir de fines de los años setenta, dada las políticas energéticas implementadas por estos países, consecuencia de los crecientes procesos de urbanización e industrialización y la conformación de amplias áreas metropolitanas iniciadas en la década de los '50s (Cf., por ejemplo, Aspelin y Santos, 1981; Balazote y Radovich, 1993; Barros, 1983; Catullo, 1986; Daou, 1985; Helm, 1982; Radovich y Balazote, 1991; Scott y Ferraz de Sá, 1991; Santos, 1983; Sigaud, 1986; Sigaud, Martins-Costa y Daou, 1987).

La instalación de grandes represas, como la de Salto Grande, es comprendida como un Proyecto de Gran Escala (PGE). Estos proyectos involucran aspectos complejos $y$ multidimensionales que comprenden factores técnicos, económicos, ecológicos, políticos y socioculturales relacionados en un intrincado juego de mutuas interacciones y condicionamientos. Por sus dimensiones, dependen, como plantea Lins Ribeiro (1987), de grandes movimientos de capital y mano de obra y engloban la presencia de un número significativo de actores sociales (Catullo, 1996:45). Entre estos actores colectivos se destacan instituciones financieras; empresas consorcistas responsables de los emprendimientos; empresas consultoras; entes gubernamentales y las poblaciones regionales y locales que ocupan las áreas destinadas a alojar las obras en cuestión (Catullo, 2006:37).

Estos proyectos presentan una lógica particular y tres dimensiones interrelacionadas: a) el gigantismo, pues son proyectos que implican grandes movimientos de capital y mano de obra; b) el aislamiento, ya que en general están ubicados en zonas aisladas, por lo que relacionan esas zonas con sistemas económicos más amplios y c) la temporalidad, es decir, el acotamiento temporal de los PGE (Lins Ribeiro, 1987:9). Los PGE se inician por la planificación, están sustentados en la racionalidad técnico- 
científica y son legitimados por discursos ideológicos basados en el progreso y el desarrollo. La conceptualización de las represas hidroeléctricas como PGE es relevante para analizar la participación de los diversos sectores involucrados pues permite contraponer a la centralización de la planificación y a los procesos de legitimación de estos PGE, la acción y la organización de las poblaciones afectadas.

A nivel local, entre los efectos resultantes de la implantación de grandes represas, se destaca la relocalización compulsiva de las poblaciones ocupantes de los espacios requeridos para la Gran Obra. Estos reasentamientos, como bien han demostrado experiencias nacionales e internacionales, establecen un acelerado proceso de modificaciones socioculturales cuyo desarrollo excede ampliamente el marco temporal demarcado por la construcción de la propia obra.

En fin, como afirma Bartolomé (1984), desencadenan por regla general, una situación de "drama social", que produce desarraigo en las poblaciones afectadas; somete a duras pruebas sus estrategias adaptativas y provoca una fragmentación de las redes de relaciones sociales (parentales, vecinales, laborales).

En este artículo nos centralizamos en cómo el género atraviesa el tema del desarrollo. Tomamos un estudio de caso (Sautu et al, 2005), es decir el proceso relocalizatorio de la ciudad de Federación ${ }^{1}$ (Argentina). En el mismo analizamos la forma desigual en que fueron afectadas las mujeres durante dicho proceso relocalizatorio. Analizamos la ciudad de Federación, sus barrios y la nueva ciudad. A partir de este trasfondo indagamos sobre la relación género-clase social y género-edad. En particular, nos basamos en los antecedentes de las investigaciones que hemos desarrollado respecto al proceso de relocalización de la ciudad de Nueva Federación desde 1979 y sistemáticamente desde

1 Denominamos Federación, al asentamiento inundado por el lago de Salto Grande; Nueva Federación (1979) a la actual ciudad, y Remanente Vieja Federación, al área no inundada del ex emplazamiento. 
1981; o sea, por más de 30 años de investigación. Es decir que hemos realizado un "Long-Term Research" (Foster et alii, 1979).

Referente a género, y siguiendo a Susana Gamboa (2008) entendemos que la "perspectiva de género", en referencia a los marcos teóricos adoptados para una investigación, capacitación o desarrollo de políticas, implica:

a) reconocer las relaciones de poder que se dan entre los géneros, en general favorables a los varones como grupo social y discriminatorio para las mujeres;

b) que dichas relaciones han sido constituidas social $e$ históricamente y son constitutivas de las personas;

c) que las mismas atraviesan todo el entramado social y se articulan con otras relaciones sociales, como las de clase, etnia, edad, preferencia sexual y religión.

\section{La represa y la ciudad de Federación}

La represa de Salto Grande, que presenta un potencial total instalado de $1890 \mathrm{Mw}$, produjo un embalse de $140 \mathrm{~km}$. de longitud y $783 \mathrm{~km}^{2}$, inundando áreas rurales y centros urbanos en el noreste de la provincia de Entre Ríos y el sudeste de la provincia de Corrientes (Argentina) y en los departamentos de Salto y Artigas (Uruguay). Su construcción se inició en el mes de abril de 1974 y sumergió la ciudad de Federación y parte del poblado de Santa Ana en la margen argentina, y parcialmente a la ciudad de Constitución y al pueblo de Belén en la margen uruguaya. Fueron relocalizadas 12.000 personas en la margen argentina y 8.000 en la margen uruguaya (Catullo, 2006:58) (Fig. 1). 
Figura 1. Localización de la represa de Salto Grande

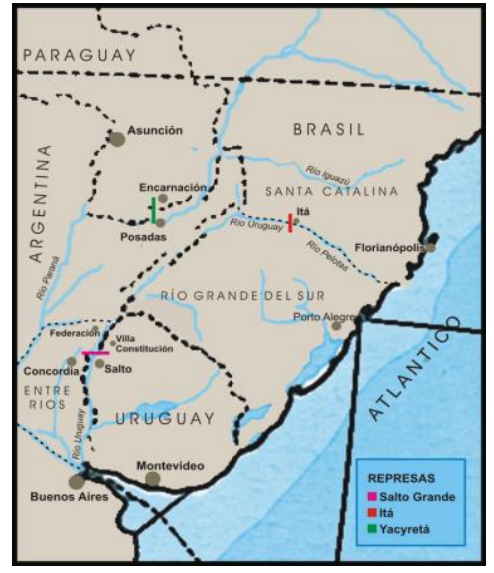

Fuente: datos propios.

En Diciembre de 1973 se firmó el Acuerdo para reglamentar el Convenio del año 1946, el cual aprobaba la construcción de la represa de Salto Grande y la planificación y ejecución de una nueva ciudad de Federación. El mismo fue aprobado por decretos de la República Oriental del Uruguay y de la República Argentina. En abril de 1974 se suscribió a su vez, un convenio entre la Comisión Técnica Mixta (CTM) (Delegación argentina), y el gobierno de la provincia de Entre Ríos, para planificar y coordinar la realización del futuro asentamiento de la ciudad de Federación. Durante el año 1975, se sancionó un nuevo Convenio entre la CTM (Delegación Argentina) y la provincia de Entre Ríos, en cuyo primer artículo esta última se hacía cargo de la ejecución de 1.019 viviendas en la ciudad Nueva Federación y la CTM se hacía cargo solamente del pago de las indemnizaciones y la provisión de fondos a la provincia, para ejecutar dichas obras. En conclusión, la planificación y ejecución de la nueva ciudad, así como del programa de relocalización estuvieron a cargo de los entes gubernamentales provinciales (Catullo, 2006: Cronograma 2:211215). 
Con referencia a la ciudad de Federación la población de la misma comenzó a ser trasladada a partir de marzo de 1979 -ya que el primero de abril de ese mismo año se inauguró la represa de Salto Grande y comenzó a funcionar la primera turbinacuando aún la ciudad no estaba concluida y dicho traslado se prolongó hasta 1981. Con respecto a las viviendas que quedaban deshabitadas en los diferentes barrios desalojados, las mismas fueron ocupadas por las familias que no tuvieron acceso a los créditos necesarios para adquirir una casa en la nueva ciudad y que formaron parte del Remanente Vieja Federación.

\section{Desarrollo, género y clases subalternas}

En muchos casos de construcción de represas hidroeléctricas no se ha tenido en cuenta a la población directa o indirectamente afectada por la misma (consultar, por ejemplo, Renk et alii, 2014) pero en el caso de la ciudad de Federación fue construida una nueva ciudad, donde se relocalizó el $70 \%$ de la población. Esto significó que no todas las familias ni las mujeres fueron afectadas de la misma manera.

Para entender mejor estas diferencias expondremos como era la ciudad de Federación, sus barrios y como los barrios periféricos fueron relocalizados en 1979 en el Remanente Vieja Federación, en casas construidas por el Instituto de Planeamiento y Viviendas de la provincia de Entre Ríos (130 viviendas) o en viviendas de los barrios periféricos que no fueron demolidas porque necesitaban usarlas para relocalizar familias de clase subalternas de Federación.

El número de viviendas que la provincia de Entre Ríos construyó en Nueva Federación fue de 1019, para lo cual tomaron los datos del Censo de Población de 1970. O sea que no se tomó en cuenta el crecimiento poblacional desde dicho año. En segundo lugar, se les dio una casa en Nueva Federación a aquellas familias que poseían trabajos formales, que pudieran pagar de su sueldo un préstamo a 30 años para adquirir una nueva vivienda. Hubo casos en que las familias más tradicionales pagaron su casa 
en efectivo. Para nuestros objetivos es relevante señalar que todas las familias que tenían trabajos informales (monteros, changarines, etc.) no pudieron acceder a una vivienda en la nueva localización $y$ en los inicios posrelocalizatorios quedaron a $36 \mathrm{~km}$ de la nueva ciudad.

Federación estaba estructurada, como la mayoría de las ciudades argentinas, sobre "el clásico trazado en manzanero legado por las Leyes de Indias" (Entre Ríos, 1973:IG 48) y fue caracterizándose de acuerdo al desarrollo de los diferentes roles económicos en la región: portuario, centro de convergencia de transporte de ferrocarril, de servicios a las colonias rurales de su área de influencia, industrial y turístico. La evolución de las funciones económicas y del accionar de las diferentes fuerzas sociales fueron determinando una particular organización del espacio urbano en el cual se distinguían diferentes "barrios" o distritos urbanos que plasmaban claramente las desigualdades de la estructura socioeconómica federaense (Catullo, 1992).

Una definición clásica de barrio que tomamos en este trabajo es la de Pierre George quien la sitúa como una unidad significativa e identititaria:

la unidad básica de la vida urbana es el barrio. Se trata a menudo de una antigua unidad de carácter religioso, de una parroquia que todavía subsiste, o de un conjunto funcional (...). Siempre que el habitante desea situarse en la ciudad, se refiere a su barrio. Si pasa a otro barrio, tiene la sensación de rebasar un límite (...) Sobre la base del barrio se desarrolla la vida pública y se articula la representación popular. Por último $-\mathrm{y}$ no es el hecho menos importante-, el barrio posee un nombre, que le confiere personalidad dentro de la ciudad (George, 1969:94).

Mientras que es Lynch quien define los distritos urbanos que conforman

secciones de la ciudad cuyas dimensiones oscilan entre medianas y grandes (...) en el que el observador entra en su 
seno mentalmente y que son reconocibles como si tuvieran un carácter común que los identifica. Siempre identificables desde el interior, también se los usa para la referencia exterior en caso de ser visibles desde afuera (Lynch, 1976:48).

Estas definiciones nos han ayudado a definir los barrios federaenses.

El Casco Central o Centro era el barrio más antiguo y el más poblado de la ciudad. Concentraba las actividades administrativas, financieras, jurídicas, recreativas y en gran medida, las comerciales. Era el lugar donde se realizaban los eventos comunitarios más importantes como la conmemoración de la Virgen Inmaculada Concepción y la Fiesta del Estudiante. Estaba habitado por familias de sectores medios y por la mayoría de los industriales y profesionales de la ciudad. En la zona Costanera se localizaban viviendas de alto costo, y en el extremo norte, el Club de Pesca y un área sobre la playa, denominada "Los Pinos", que conformaba el centro de reunión de mujeres y varones federaenses durante la época estival. Existían dos clubes deportivos -exclusivos para varones-, donde se practicaba fútbol, el Club Adelante Juventud, donde concurrían los adolescentes asociados al Club Social Federación y el Club Estudiantes, donde practicaban deporte los jóvenes de los sectores socioeconómicos de menores recursos. En síntesis, el Casco Central representaba el origen de la ciudad y se comportaba como el corazón de la misma. Excepto una "confitería", la plaza y "Los Pinos", lugares de reunión de adolescentes y jóvenes de ambos sexos, las mujeres sólo concurrían a la Iglesia.

Al norte de la planta urbana, y también lindando con el río, se situaba el barrio Industrial. En su inicio fue un barrio de varones. Nacido por la necesidad de recibir y trabajar la madera que llegaba en jangadas desde el sur de Brasil y desde la provincia de Misiones (Argentina), y que tenía como punto terminal la zona norte de Federación, fue desarrollándose con la instalación de aserraderos y de familias cuya fuente de trabajo era la actividad maderera (Entre Ríos, 1974). 
Hacia el oeste de la ciudad, desde las vías del ferrocarril, se extendía el barrio San Lorenzo. De formación más reciente (19531955), provenía de loteos efectuados por familias tradicionales de Federación y cuyos compradores fueron, fundamentalmente, colonos y sus familias provenientes del ejido y familias obreras de las industrias locales (Entre Ríos, 1974). Típico barrio de obreros, sus elementos más característicos eran un centro comercial que competía con el área comercial del Casco Central y el Club Atlético San Lorenzo -el club deportivo más importante de la ciudad-. Sus viviendas, de construcción más reciente y más económicas que las del Casco Central, contaban con huertas y criaderos de aves a cargo de las mujeres. Se había constituido en el sector más dinámico de la ciudad. Al principio, cumplió como rol fundamental alojar a la población obrera de los aserraderos, pero paulatinamente fue adquiriendo fisonomía propia y comenzó a competir con el Casco Central el cual mantuvo siempre su supremacía a nivel administrativo, financiero y político.

Lindando con el barrio San Lorenzo, hacia el noroeste de la ciudad, se ubicaba el barrio Salto Grande. Construido en los años 1968-69, a través de un plan de viviendas con participación de recursos nacionales, provinciales y municipales, estaba habitado mayormente por familias obreras y representaba una zona satélite del barrio San Lorenzo.

El barrio San Miguel, construido por el mismo plan que el barrio Salto Grande, se situaba hacia el sur de la ciudad, separado del Casco Central por las vías del ferrocarril. Así denominado por la capilla homónima, lo habitaban familias de obreros y empleados y empleadas públicos y conformaba un área dependiente del Casco Central.

Los barrios Miguelito y La Virgen fueron construidos a fines de la década de los setentas, por planes municipales de vivienda para realojar a familias que vivían en casillas muy precarias sobre terrenos fiscales o privados, dispersas en diferentes áreas de Federación. En estos barrios habitaban los sectores subalternos de 
Federación, es decir, las familias de los obreros de los aserraderos, de los changarines urbanos, de los zafreros y los "monteros". ${ }^{2}$ Las viviendas eran de ladrillos, de terminación precaria y poseían un amplio terreno donde se ubicaban los sanitarios, la huerta familiar y el criadero de aves a cargo mayormente de las mujeres. La diferencia que presentaban entre sí era que, mientras el barrio Miguelito actuaba como área satélite del Casco Central, el barrio La Virgen lo era respecto al barrio San Lorenzo. Las mujeres solían trabajar en la limpieza de casas de familias de clase media (Fig.2).

Figura 2- Planta urbana Federación

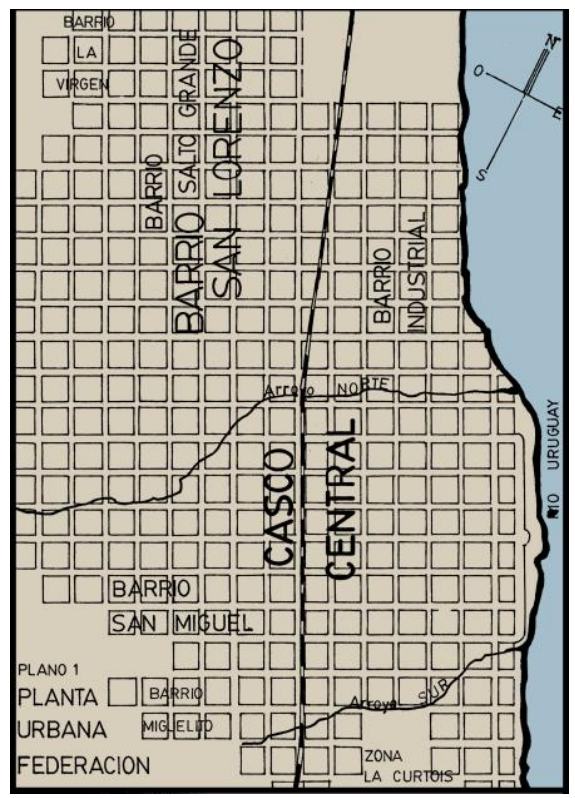

Fuentes: Municipalidad de Federación y datos personales.

\footnotetext{
2 Los "monteros" son jornaleros que talan los montes de madera necesarios para los aserraderos locales. Generalmente, de lunes a viernes se instalan en los montes, durmiendo en casas muy precarias hechas con restos de madera, en otras ocasiones son llevados cada madrugada en camiones al monte. Sus mujeres quedan en sus casas cuidando de sus hijos y ancianos, y de la huerta y el criadero de aves.
} 
En general, en el período previo a su desmantelamiento, en la fisonomía extendida de Federación, de construcciones bajas - de no más de dos niveles-, y muy abundante vegetación, solamente se destacaba en altura el volumen de la torre de la Iglesia. Calles amplias, veredas anchas, y en definitiva un espacio público de generosas proporciones que delineaban una imagen urbana chata, semejante en el centro y en los barrios (Patti y Catullo, 2001:110111).

Como consecuencia de la formación del lago de Salto Grande, en Federación se inundaron la zona Costanera y el Casco Central, es decir, el área con mejor infraestructura, mayor equipamiento y donde vivían las familias tradicionales de la ciudad. Quedaron bajo las aguas la sede de la Gendarmería; la plaza "Nueve de Julio"; la Jefatura de Policía; la Intendencia; la Iglesia "Inmaculada Concepción"; la Dirección Departamental de Escuelas; el Juzgado de Paz; el Correo; la Central Telefónica; el Museo Regional; la Escuela $n^{\circ} 1$ y las escuelas privadas; el Colegio Secundario; los bancos; los comercios más tradicionales y especializados, las "confiterías" céntricas; el Club Social Federación; el Club Estudiantes; el Cine Rex; la mayoría de los consultorios de los profesionales. También, se inundaron el barrio Industrial, donde estaban asentados el mayor número de aserraderos y la curtiembre, y las quintas de fin de semana, situadas en la zona La Curtois. La estación de trenes también quedó bajo las aguas y, en el barrio San Lorenzo, si bien fue afectado parcialmente, se inundaron la mayoría de sus comercios; la escuela $n^{\circ} 42$ y el Club Atlético San Lorenzo.

Respecto a los barrios Salto Grande y San Miguel -donde se situaba las capillas San Miguel y Cristo Obrero y el Hospital San José- fueron parcialmente afectados. Por último, los barrios periféricos fueron afectados en forma diferencial. El barrio La Virgen fue totalmente inundado por su cercanía con el arroyo homónimo; en tanto que en el barrio Miguelito la mayoría de las viviendas quedaron en pie.

En síntesis, en la ciudad de Federación, el lugar de encuentro cotidiano de las mujeres era el barrio, sus vecinas, y los 
lugares de compras diarias o semanales. Además, sus lugares de trabajo en el caso de las maestras y algunas empleadas públicas. Referente a los lugares de recreación sólo estaba la confitería "La Ideal", durante las horas diurnas, que se situaba en una esquina, frente a la Plaza "9 de Julio", la Plaza misma y el Club Social Federación, pero sólo para reuniones de las clases sociales más tradicionales de Federación.

\section{La plasmación del Proyecto: la ciudad Nueva Federación}

La nueva ciudad tuvo una propuesta arquitectónica muy diferente de la anterior. Esta propuesta fue delineada durante el gobierno dictatorial (1976-1983) y se recostó en una concepción teórica que omitió las particularidades que definían lo singular y lo propio de la población federaense y su hábitat, en particular para la mujer, que vio transformada su ciudad, su barrio y su vivienda.

El proceso que condujo a la construcción efectiva de Nueva Federación se asentó sobre ciertos antecedentes. Estos se caracterizaron por continuas restricciones, cada vez mayores, en la disposición de recursos financieros para toda acción destinada a resarcir los daños infringidos al área de influencia de la construcción de la represa de Salto Grande y a las poblaciones afectadas por la misma. La ciudad Nueva Federación siempre fue considerada por las autoridades de la Comisión Técnica Mixta de Salto Grande y por los gobiernos nacionales, en especial por los gobiernos militares, o sea, a partir de 1976, un hecho secundario respecto al Proyecto de Gran Escala, de gran interés nacional.

Al concluirse la etapa de proyecto, durante el proceso de contratación y ejecución de las obras de la nueva ciudad, se operaron una serie de modificaciones restrictivas en la trama urbana por parte de los gobiernos nacionales y provinciales de facto. Dichos recortes se constituyeron en hechos objetables que aportaron nuevos obstáculos y condicionamientos negativos respecto de la calidad de vida de las familias y de las mujeres de Federación. 
Con respecto al centro urbano, la concentración de funciones públicas y sociales -que los proyectistas dispusieron en un eje troncal paralelo a la costa del lago, distribuidas en los centros cívico, cultural-deportivo y turístico-, sufrió una fuerte variación en la ejecución de las obras, al eliminarse algunos edificios, tales como la Municipalidad, la Dirección Departamental de Escuelas, el centro cultural-deportivo en su totalidad, $e$ implementarse la ubicación de otros en cualquier lugar de la estructura urbana distinto al que había sido previsto, en gran medida, consecuencia de las exclusiones antes detalladas. De este modo, con la desaparición de ciertas funciones que se desenvolvían en la vieja ciudad se suprimió la posibilidad de satisfacer necesidades ya existentes -con las consecuencias que una decisión como esa implica para la población la cual estaba muy estresada por la desinformación y los rumores de estos cambios que si fueron conocidos por el poder Ejecutivo Local- y con el traslado de otras, se desarticuló la afinidad funcional que se había intentado generar entre ellas, quedando libradas a su suerte potenciales situaciones de conflicto. Esto generó, por ejemplo, que durante muchos años no existiera una plaza principal, teniendo en cuenta el rol social que cumplía en la antigua ciudad, lugar de encuentro de amigas; de parejas de novios, tanto como de familias con sus hijos. La actual Plaza "Libertad" se inauguró recién en el año 1991.

Al materializarse la construcción de la ciudad, dificultades muy concretas fueron las que produjeron la supresión de algunos aspectos de la infraestructura de servicios domiciliarios. Por ejemplo, la red urbana de suministro de gas se eliminó y se instalaron cocinas y calefones eléctricos en las nuevas viviendas, con el argumento falaz de que la proximidad de la represa de Salto Grande garantizaría a la población federaense energía eléctrica a muy bajo costo. Lo cierto es que las facturas domiciliarias por consumo eléctrico promediaban en 1982 el monto de un salario mínimo. Situación que las usuarias se vieron obligadas a revertir a su propia costa, instalando sistemas de gas envasado con el consiguiente cambio de artefactos, y otras, 
volviendo a los "tradicionales" cocina y horno de barro con uso de leña. La Zona Industrial, que en el proyecto original fue localizada en el borde externo de la ciudad, paralela a la costa separada por una reserva forestal, en los hechos no se incorporó a su traza sino que quedó en el Remanente Vieja Federación, inicialmente a unos 36 kilómetros de la nueva ciudad. Distancia que por largo tiempo careció del actual puente de 5 kilómetros que une ambos emplazamientos -que fuera inaugurado en el año 1985-, y de un transporte adecuado para cubrirla, generando un grave problema para quienes cotidianamente debían recorrerla para ir a trabajar. En consecuencia, los industriales locales tuvieron que colocar camionetas para trasladar a sus obreros al nuevo Parque Industrial, pero no fue así para las maestras, enfermeras y mujeres que debían cruzar en pequeños e inseguros botes el lago de Salto Grande o caminar hasta el Remanente Vieja Federación. En la actualidad ya no funciona ninguna escuela en dicho Remanente, salvo un área de la municipalidad cuyos empleados se trasladan en automóviles.

El Remanente Vieja Federación, que constituye el área no inundada del anterior asentamiento, presentó una trama desdibujada por los vacíos existentes entre las diversas edificaciones que se han ido colmando de vegetación. En los primeros años posrelocalización estuvo conformada por la Zona Industrial; por un grupo de unas 130 viviendas económicas construidas en 1978 por el gobierno de la provincia de Entre Ríos; por viviendas donde relocalizaron a las familias de sectores subalternos, pertenecientes a diferentes barrios de Federación; por construcciones no demolidas, como el antiguo hospital que cobijaba el asilo de ancianos y por nuevas edificaciones, como una escuela primaria y una parroquia, a los que se debe sumar el cementerio que no fue relocalizado. De esta forma, la materialización del proyecto de una nueva ciudad tuvo como resultado una ciudad partida en dos: Nueva Federación o "La Nueva" y el Remanente Vieja Federación o "La Vieja", donde habitaron los sectores subalternos de la sociedad federaense (Fig. 3). Lo significativo para nuestros objetivos es que la mayoría de las 
mujeres de los sectores subalternos que trabajaban en casas de familia realizando limpieza por horas, no podían dejar a sus hijos solos, ya que a esto debemos sumar que se desdibujaron las redes de relaciones parentales $y$ vecinales, perdiendo una fuente importante de dinero para ellas y su familia. Además, las viviendas fueron construidas sobre un suelo muy pedregoso que les hacía imposible volver a desarrollar sus huertas. Durante estos primeros años se dedicaron a la reproducción y cuidado de la familia, sin posibilidad de poseer un trabajo remunerado.

Figura 3. Nueva Federación y Remanente Vieja Federación. Años ' 80

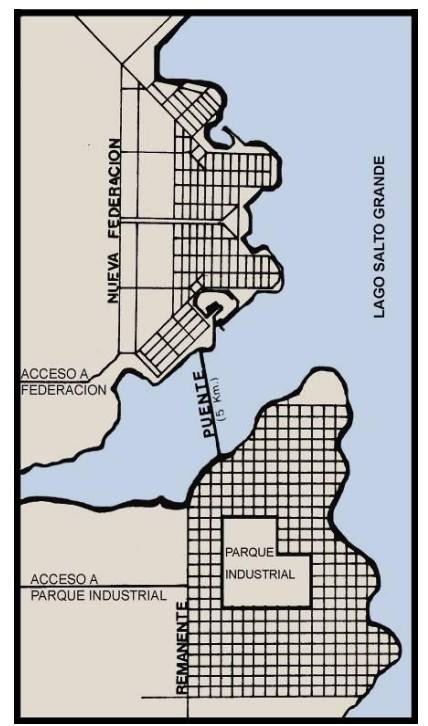

Fuente: Municipalidad de Federación

\section{El territorio fragmentado: La Nueva Ciudad y el Remanente Vieja} Federación

Las familias federaenses y las mujeres quedaron, como hemos ya expuesto, divididas entre las de "la Vieja" y las de "la Nueva", entre ellas y nosotras. Donde en los primeros veinte años de la relocalización en "la Nueva" vivían los sectores medio-altos y 
medios, y en "la Vieja" los sectores bajos que formaron barrios, como los denomina Gravano (2003:24-25) negros (como los films) con imágenes negativas y estigmatizantes de parte de los habitantes de la nueva ciudad quienes defendían su blancura ${ }^{3}$ Así, el imaginario de éstos los llevaba a decir que las familias más pobres "sacaban los inodoros del baño o levantaban los pisos de las nuevas casas" y que en "la Vieja" se veían mujeres y hombres a los costados de las calles tirados por la borrachera. Pero los tranquilizaba que estaban del otro lado del brazo del lago de Salto Grande. Esta es una percepción que, como vemos, no sólo afecta a las mujeres; pero, sin duda alguna, para las clases medias federaenses el pensar en una mujer ebria es mucho peor que pensar en un hombre.

En la actualidad, en Nueva Federación se han construido muchos barrios con fondos nacionales y provinciales (Fig.4). Nos enfocamos en el Barrio CAFESG, así llamado por los federaenses ya que fue construido en etapas, con las regalías de la represa de Salto Grande, por la "Comisión Administradora de los Fondos Especiales de Salto Grande" (CAFESG) que fuera creada por el Gobierno de la Provincia Entre Ríos en el año 1998. La CAFESG arregló otros barrios precarios de Nueva Federación, pero, en nuestra opinión y basándonos en nuestros trabajos de campo, el barrio CAFESG ha sido el más relevante, ya que presenta 603 viviendas que comenzaron a construirse en el año 2000, y porque fue producto de una decisión política provincial para el "resarcimiento por el traslado que no tuvieron aquellas familias que quedaron en el Remanente Vieja Federación". Esto nos lleva a demostrar que hubo 2 grupos diferentes de mujeres. Aquellas que fueron relocalizadas a la nueva ciudad entre abril de 1979 y marzo de 1981, y otras, más de 600 que fueron relocalizadas en el propio remanente en el año 1979 en viviendas no demolidas, que

3 Gravano explica la "blancura" de un barrio cuando sus propios habitantes dicen ser honestos y trabajadores, por ejemplo. O sea, hablan muy bien del barrio (2003:22-24). 
sufrieron la exclusión de la infraestructura y equipamiento de la nueva ciudad, y de los trabajos formales e informales generados en Nueva Federación. Ellas y sus hijas -ya que los varones generalmente trabajan en los aserraderos de la Zona Industrial o en la construcción- durante muchos años tuvieron que caminar los $5 \mathrm{~km}$ del puente que une "la Vieja" y "La Nueva", a lo que hay que sumarle que el nuevo emplazamiento tiene $7 \mathrm{~km}$ de longitud, para poder conseguir trabajos en casas de familias, su mayor entrada de dinero. Y de esta manera, esas mujeres vivieron dos veces el Stress Multidimensional de Relocalización (Scudder y Colson, 1982); la desinformación referente a qué vivienda les tocaría, en qué lugar, y la consecuente pérdida de su red vecinal y parental.

Además, es importante señalar que el barrio CAFESG forma parte de una zona de barrios periféricos, de "viviendas sociales", realizadas con fondos de la provincia de Entre Ríos, la Nación y el municipio que han crecido en la nueva ciudad y que no forman parte de la zona planificada (1979) ni de la Zona Turística (1997) (Finvbarb y Masetto, 2011), y que son prejuzgados por los sectores medios como violentos, como barrios negros al decir de Gravano (2003:24-25), donde hay drogadictos, drogadictas, "cocinas de droga", adolescentes embarazadas que cobran un Plan Nacional de Ayuda, de donde "salen delincuentes en moto" que roban en el Centro de la ciudad y en la zona turística termal, formada desde el año 1997 cuando se desarrolló el Parque Termal Municipal, que está llena de hoteles, bungalows, restaurantes, etc. visitados por turistas de todas partes del país. Esta zona es denominada por los sectores medios como la "Zona de los barrios", escasamente conocida por los habitantes de la zona planificada. 
Figura 4. Nueva Federación y Remanente Vieja Federación.

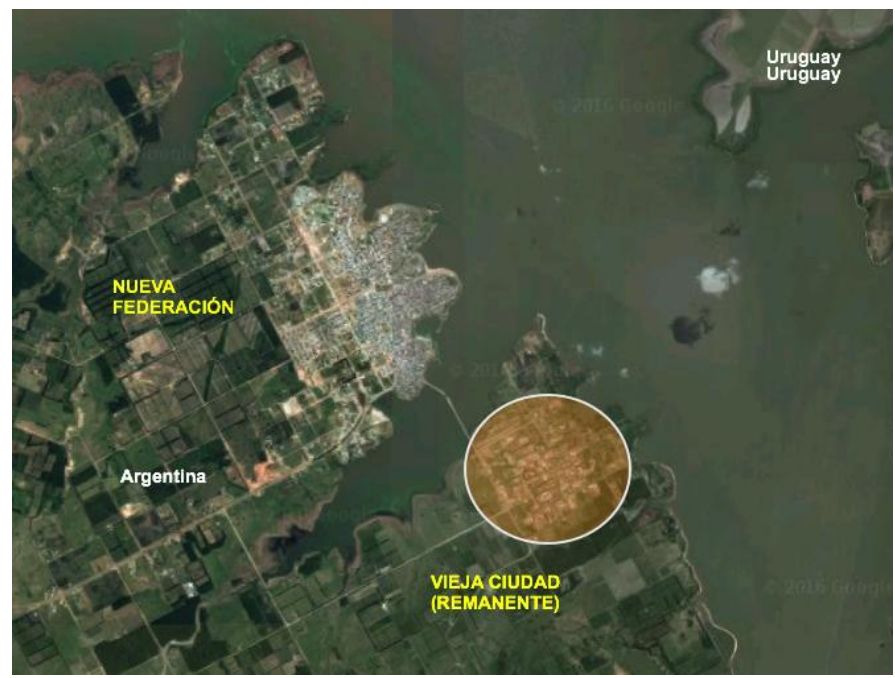

Fuente: Google Earth. Acceso: 18/5/2017.

\section{La desarticulación de los barrios y las mujeres}

En principio destacamos que el proceso relocalizatorio de la comunidad federaense presentó características especiales ya que generalmente cuando se relocaliza un sector urbano -como es el caso de la población de la ciudad de Posadas afectada por la construcción de la represa binacional argentino-paraguaya de Yacyretá- los otros componentes urbanos (de infraestructura, de equipamiento y humanos) mantienen sus espacios dentro de la ciudad; no fue así en el caso de Federación donde se modificó no sólo la estructura urbana sino también la ubicación de las mujeres dentro de esa nueva organización espacial. Esto produjo una desarticulación del espacio físico, la pérdida de la identidad barrial y la pérdida de la imagen de la mujer federaense respecto de su ciudad. Asimismo, el diseño de la nueva ciudad, tal como explicamos anteriormente, tradujo la falta de conocimiento y de consideración por los valores culturales y sociales de las familias federaenses como bien hemos señalado junto con la arquitecta 
Patti (2001). La mujer que habitó Nueva Federación experimentó, por lo tanto, y especialmente en los primeros años después del traslado, la pérdida del ambiente cotidiano que había recreado día a día, en particular en su vivienda, en su patio, en su jardín. Esa alteración de la organización espacial desarticuló a su vez las relaciones, es decir aquellas que llevan consigo el intercambio de servicios, información y aprobación personal entre aquellas que viven, unas cerca de otras, y que habían estado tan consolidadas en la antigua trama urbana. Se produjo entonces una dispersión de las unidades barriales constituidas a través de muchos años de convivencia diaria.

Por supuesto que también las mujeres relocalizadas en el remanente se vieron afectadas por la desarticulación de sus vecindarios y de las asociaciones barriales, $\mathrm{y}$, al mismo tiempo, por la dispersión de sus familias extensas ya que algunos de los miembros del grupo familiar pudieron acceder a la posesión de una vivienda en la nueva ciudad, debilitándose de esta manera las relaciones parentales con aquellos que permanecieron en el antiguo emplazamiento. Ello provocó una continua añoranza por el anterior vecindario y una actitud de desconfianza y recelo ante las nuevas vecinas.

La falta de comunicación con las vecinas en los diferentes contextos espaciales, como por ejemplo la calle, los comercios diarios, afectó ese intercambio de servicios, de información que se había construido a través del tiempo y que conformaban parte de la vida diaria de las mujeres federaenses.

\section{Género, Edad y Procesos de Relocalización}

En este apartado, tomamos un trabajo pionero para las relocalizaciones de población por Proyectos de Gran Escala, como es el de Scudder y Colson (1982:273) quienes fueron los primeros en concebir a las relocalizaciones como procesos y destacaron en sus trabajos que las respuestas de los grupos afectados por estos procesos se diferencian según tres variables: 1) género, 2) edad y 3) posición dentro del sistema de estratificación social, para destacar la relación 
género y edad. En nuestros trabajos de campo pudimos comprobar que las mujeres que más sufrieron la relocalización, fueron aquellas mayores de 50 años, en particular las que vivían en la zona central de la ciudad de Federación. Tanto en entrevistas personales como en entrevistas a médicos de la ciudad Nueva Federación y de la ciudad de Concordia, que se sitúa a $50 \mathrm{~km}$ al norte de la actual ciudad, hemos constatado una mayor morbilidad (depresión, infartos, por ejemplo), lo que se denomina el "síndrome de la casa perdida", es decir, una gran nostalgia por la anterior vivienda, sus espacios, sus plantas, sus mascotas. Y es interesante que, aún hoy, haya muchas mujeres que continúan diciendo que hubo personas que "murieron de tristeza" por el desarraigo sufrido en una ciudad que desconocían; que estaba en plena construcción y que no tenían plantas ni árboles... una verdadera "maqueta".

\section{La falta de vida comunitaria}

En los primeros años postrelocalización, hubo una ausencia de instituciones barriales y una carencia de espacios privados de uso social donde se generaban y estimulaban las relaciones interbarriales y que en el anterior emplazamiento estaban representados por las confiterías, donde se juntaban las jóvenes de clase media y también los bares, que era un "terreno" sólo de varones.

Las mujeres federaenses habían perdido sus lugares, aquellos rincones que favorecían la cohesión social, que extendían la red de relaciones sociales, donde diariamente se protagonizaban hechos que luego quedaban registrados en la memoria colectiva.

Así, durante la etapa que prosiguió inmediatamente al traslado, reinaban la apatía, la abulia puesto que cada una debía hacer frente a su propio "drama". No sólo las relaciones intracomunitarias se vieron realmente afectadas por la relocalización sino que también se resquebrajaron las relaciones extracomunitarias quedando Nueva Federación/Remanente y Vieja Federación casi aisladas del contexto regional. 
La nueva ciudad se había transformado, tal como adelantáramos, en una especie de "gran maqueta" desarticulada, donde cada familia y cada mujer debió desarrollar sus propios dispositivos de adaptación ante la modificación tanto de medio ambiente natural como social.

\section{Consideraciones finales}

En primer lugar, queremos destacar que la relocalización de la ciudad de Federación fue la primera relocalización urbana de Argentina, por lo tanto una experiencia única, un "field" para diferentes abordajes desde la Antropologia Social y otras Ciencias Sociales.

Por otra parte, no quisimos tratar el tema de género y toma de decisiones del proceso de relocalización, pues este fue nulo. Y no hubo organización alguna por parte de las mujeres ante las grandes decisiones (por ejemplo, valuaciones e indemnizaciones de las viviendas por parte de la Comisión Técnica Mixta; estándar de vivienda y ubicación de la misma en la futura ciudad), pero si podemos confirmar que han sido las mujeres quienes han producido las modificaciones posteriores en nuevas casas, para mejorar la calidad de vida de su familia y de ellas mismas. Además, ayudaron a sus maridos en sus emprendimientos económicos. Y con el tiempo, ellas mismas son, en la actualidad, emprendedoras, comerciantes. En los primeros tiempos postraslados lo primordial para las mujeres fue su espacio cotidiano, su familia nuclear, su familia extensa.

Como explicitáramos, las mujeres de clase media perdieron las redes de relaciones sociales, la convivencia con sus vecinas, mientras que en el caso de las mujeres de sectores subalternos perdieron, además de sus relaciones vecinales y parentales, sus fuentes de trabajo.

Asimismo, intentamos, tomando como trasfondo los cambios de la ciudad de Federación, centralizarnos en aquellos temas de los cuales poseemos más datos: la desigualdad entre las mujeres de sectores medios y aquellas de los sectores subalternos, 
y el sufrimiento de las mujeres de mayor edad frente a un cambio de tal magnitud.

Consideramos importante resaltar que hemos leído diferente bibliografía sobre género, y género y desarrollo, tales como Kate Young (1997), Gita Sen (1996), Lourdes Beneria y Gita Sen (1982), Marta Lamas (2008), Nidia Pesántez (2007), o Carla Zumbado (2003) quienes analizan las posturas y los movimientos teóricos sobre género y desarrollo pero dimos mayor importancia a los datos de nuestra investigaciones, antes que presentar análisis teóricos sobre género pero que sí tuvimos en cuenta en nuestro trabajo. Señalamos, por último, retomando a Gita Sen, que "el empoderamiento económico es a menudo la llave para romper el cruel ciclo de la desigualdad de género" (1995:61) en particular, en los Grandes Proyectos de desarrollo que son nuestro objeto de estudio.

\section{Referencias bibliográficas}

Aspelin, Paul; CoelHo dos Santos, S. The Indian Areas Threatened by Hydroelectric Project in Brazil. IWGIA (44), Copenhagen, 1981.

BAlAZOTE, Alejandro; RADOvich, Juan C. Gran obra e impacto en Pilquiniyeu. CEAL (438), Buenos Aires, 1993.

BARroS, H. O. M. Projeto Sobradinho: avaliação sócio-ecônomica da relocalização populacional. Informe de Investigación, CHESF/Fundação Joaquim Nabuco/Instituto de Pesquisas Sociais. Recife, 1983.

BENERIA, Lourdes; SEN, Gita. Acumulación, reproducción y el papel de la mujer en el desarrollo económico: Una revisión a Baserup. In: LEÓN, Magdalena (comp.). Debate sobre la mujer en América Latina y el Caribe: discusión acerca de la unidad producciónreproducción. Bogotá, ACEP, 1982, pp.23-39.

CATULLO, María Rosa. Relocalizaciones compulsivas de población: estudio de un caso. Ciudad Nueva Federación, Entre Ríos. Runa (XVI), Instituto de Ciencias Antropológicas, Universidad de Buenos Aires, Buenos Aires, 1986, pp.137-156. 
- Reconstrucción de la identidad y Proyectos de Gran Escala: Ciudad Nueva Federación, Provincia de Entre Ríos, Argentina. Série Antropología, (125), Universidad de Brasilia, 1992.

. Poder y Participación en Proyectos de Gran Escala. Análisis comparativo de los procesos de relocalización por la construcción de la represa binacional argentino-uruguaya de Salto Grande. Tesis (Doctorado en Estudios Comparativos sobre América Latina y el Caribe), Facultad Latinoamericana de Ciencias Sociales (FLACSO) y Universidade de Brasília (UnB), Brasília, 1996.

Ciudades Relocalizadas. Una mirada desde la Antropología Social. Buenos Aires, Editorial Biblos, 2006.

DAOU, Ana Maria Lima. A intervenção do Estado e o reassentamento em núcleos rurais no caso de Sobradinho: reestruturaçâo da comunidade camponesa. Proyecto de Tesis de Maestría, Programa de Pósgraduaçâo em Antropologia Social, Museu Nacional, UFRS, Rio de Janeiro, 1985.

ENTRE Rios. Federación. Proyecto Nueva Ciudad, Primer proyecto. Instituto Autárquico de Planeamiento y Vivienda, Paraná, 1973.

. Informe de Relocalización. Estudio Nueva Federación y Santa Ana (ENFYSA), Paraná, 1974.

FINVBARB, Mariel; MASETTO, Daniella. Los aportes de la urbanística en la construcción de la Identidad Social Urbana de la ciudad planificada de Nueva Federación. Tesina de Grado, Facultad de Arquitectura, Universidad Nacional del Litoral, Santa Fé, 2011.

FOSTER; George et alii. (ed.). Long-term Field Research in Social Anthropology. N. York, Academic Press, 1979.

GAMBOA, Susana. ¿Qué es la perspectiva de género y los estudios de género? Mujeres en red. El periódico feminista, (s/d), 2008 [http://www.mujeresenred.net/spip.php?article1395 - acceso 11 mayo 2017].

George, Pierre. Geografía Urbana. Edit. Ariel, Barcelona, 1969.

Gravano, Ariel. Antropología de lo Barrial: estudios sobre producción simbólica de la vida urbana. Editorial Espacio, Buenos Aires, 2003. 
HeLm, Cécilia M. Vieira. A terra, a usina e os índios PI Mangheirinha. In: SANTOS, Silvio C. dos (org.). O índio perante o direito. Ed. da UFSC, Florianópolis, 1982, pp.129-142.

LAMAS, Marta. El género es cultura, (s/d), 2007 [http://www.oei.es/euroamericano/ponencias_derechos_genero.php acceso 5 mayo 2017].

LYNCH, Kevin. La imagen de la ciudad. Ediciones Infinito, Biblioteca de Planeamiento y Vivienda, vol. 9, Buenos Aires, 1976.

PatTi, Beatriz; CATullo, María Rosa. Proceso de relocalización y Nueva ciudad: Federación-Nueva Federación, Entre Ríos, Argentina. In: Balazote, Alejandro; Radovich, Juan Carlos; Catullo, María Rosa (org.). Antropologia y Grandes Proyectos en el Mercosur. Editorial Minerva, La Plata, 2001, pp.107-126.

PESÁNTEZ, Nidia. De la teoria a la práctica. Un paso adelante en la transversalización de género. Quito, PNUD, 2007.

RADOVICH, Juan Carlos; BALAZOTE, Alejandro. La etnicidad mapuche en un contexto de relocalización: la represa de Piedra del Aguila. América Indígena, vol. LI (1), México, 1991, pp.277-319.

Renk, Arlene; Agnolin, G. L.; WinckLeR, Silvana. Como peixes fora d'água, o caso dos pescadores profissionais artesanais na UHE Foz de Chapecó. Revista Antropolítica (37), Niteroi, 2 sem., 2014, pp.137156.

RIBEIRO, Gustavo Lins. ¿Cuánto más grande mejor? Proyectos de Gran Escala: una forma de producción vinculada a la expansión de sistemas económicos. Desarrollo Económico (105), vol. 27, Ediciones del IDES, Buenos Aires, 1987, pp.3-28.

SANTOS, Silvio Coelho dos. Construção de barragens e sociedades indígenas no sul do Brasil. In: América Indígena, vol. XLIII, (2), México, 1983, pp.319-342.

SATRIANI, L. M. Lombardi. Antropología Cultural. Análisis de la cultura subalterna. Galerna, Buenos Aires, 1975.

SAUTU Ruth, et alii. Manual de Metodología. CLACSO Libros. Buenos Aires, 2005. 
SCOTT, Parry R.; FERRAZ DE SÁ, María A. Barragem de Itaparica: Indagações sobre a construção de base familiar da produção agrícola. Trabajo presentado en el Seminário sobre Grandes Projetos, Belém, UFPe/Naes, 1991.

SCUDDER, Thayer; Colson, Elizabeth. From Welfare to Development: a Conceptual Framework for the Analysis of Dislocated People. In: ART, Hansen; OlIVER-SMITH, Anthony (comp.). Involuntary Migration and Resettlement. The Problems and Responses of Dislocated People. Westview Press, Boulder, Colorado, 1982, pp.267-287.

SEN, Gita. Una economía alternativa desde la perspectiva de género. In Development: Journal of the Society International Journal (SID), 1995, pp.1-65.

SigAud, Lygia. Efeitos sociais de grandes projetos hidrelétricos: as barragens de Sobradinho e Machadinho. Comunicação $\mathrm{N}^{\circ}$ 9, Programa de Pós-graduação em Antropologia, Museu Nacional, UFRJ, Rio de Janeiro, 1986.

YOUNG, Kate. El potencial transformador en las necesidades prácticas: Empoderamiento colectivo y el proceso de planificación. In: LEÓN, Magdalena. Poder y empoderamiento de las mujeres. TM Editores, Facultad de Ciencias Humanas, Bogotá, 1997, pp.121-129.

ZUMBADO, Carla. Género y Políticas de Desarrollo: la brecha entre el decir y el hacer. Desarrollo rural y políticas agropecuarias en Costa Rica. Tesis (Doctorado en Ciencia Política y Derecho Público), Universitat Autónoma de Barcelona, 2003. 